\title{
Approximate solution for pressure behavior during a multiple rate injectivity test
}

\author{
Maíra Fortes Bonafé ${ }^{*}$ Arthur Braga' ${ }^{1}$ Abelardo Borges Barreto Jr. ${ }^{2}$
}

Received: 20 July 2019 / Accepted: 6 March 2020 / Published online: 16 March 2020

(c) The Author(s) 2020

\begin{abstract}
The pressure response during a well test provides important information about reservoir properties, well productivity or reservoir performance. In order to have an option to replace traditional production tests, avoiding the gas flaring and increasing operational safety, injectivity test began to play an important role in the management of reservoirs. Although analytical models are able to describe injection and falloff periods, multiple rate models still need to be developed. This work presents a new formulation to compute pressure response when performing multiple rate injectivity test in a water injection well operating in an oil zone. First, the proposed formulation is compared to injection/falloff models and after a multiple rate scheme is performed. This formulation extends prior models presented in the literature. The accuracy of the proposed solution was assessed by comparison with a numerical simulator. The proposed formulation is also used to determine the reservoir equivalent permeability in any specific period of injection or shut-in.
\end{abstract}

Keywords Well-testing $\cdot$ Injectivity test $\cdot$ Partial differential equation $\cdot$ Reservoir evaluation

\section{List of symbols}

$B \quad$ Phase formation volume factor

$c_{\text {to }} \quad$ Total compressibility

$c_{\mathrm{r}} \quad$ Rock compressibility

$f_{\mathrm{w}} \quad$ Fractional flow of water

$h \quad$ Thickness (m)

$k_{l} \quad$ Absolute permeability $(=o$,

$k_{\mathrm{r} l} \quad$ Relative permeability $(=$,

$M \quad$ Mobility ratio

$p_{\mathrm{i}} \quad$ Initial pressure

$p_{l} \quad$ Pressure $(=, w)$

$p_{\text {wf }}$ Well bottom-hole pressure

$q_{\text {inj }}$ Injection rate

$q_{\mathrm{o}} \quad$ Oil rate

$q_{\mathrm{w}} \quad$ Water rate

$r_{\mathrm{f}} \quad$ Waterfront radius

$r_{\mathrm{w}} \quad$ Wellbore radius

$S_{\mathrm{w}} \quad$ Water saturation

$t \quad$ Time

Abelardo Borges Barreto Jr.

abelardo.puc@gmail.com

1 Mechanical Engineering Department, PUC-Rio, Rio de Janeiro, Brazil

2 Mechanical Engineering Department, Mathematics Department, PUC-Rio, Rio de Janeiro, Brazil

$\begin{array}{ll}\alpha_{t} & 19.03 \\ \alpha_{t} & 0.0003484 \\ \gamma & \text { Euler constant } \\ \mu_{l} & \text { Viscosity of phase }(=,) \\ \lambda_{l} & \text { Mobility of phase }(=,) \\ \lambda_{\mathrm{t}} & \text { Total mobility } \\ \varphi & \text { Porosity }\end{array}$

\section{Introduction}

Flaring gas wastes a valuable energy resource that could be used to support economic growth and progress. It also contributes to climate change by releasing millions of tons of $\mathrm{CO}_{2}$ to the atmosphere. To reduce gas flaring and increase operational safety, solutions such as the re-injection production tests, closer chamber and injectivity tests have been highlighting.

In an injectivity test, a single phase (water) is injected for a certain period into an oil-saturated reservoir and then the well is shut, beginning a period of falloff, when, consequently, a zero-rate pulse propagates along the reservoir. Reservoir parameters, permeability and skin can be obtained from the study of the behavior of well bottom-hole pressures during the injectivity tests. In addition, it is possible to 
evaluate the reservoir connectivity and the injection potential of the well.

Thompson and Reynolds (1997) provided a general theory for the pressure behavior in infinite radial heterogeneous reservoirs under multiphase flow, including the injectivity test problem.

Peres et al. (2003) extended these concepts to develop analytical solutions for the pressure behavior during the falloff period, after a period of water injection. Both solutions can be used to determine permeability and skin factor in an infinite acting radial reservoir.

Approximate analytical solutions for the injection pressure response at a horizontal water injection well have been presented by Peres et al. (2004), using Buckley and Leverett's (1942) solutions to the water saturation profile based on the proposed models for water displacement.

Subsequently, Peres and Reynolds (2006) used the superposition principle to provide a solution in the falloff period. Although the injection problem was nonlinear, these authors relied on the work of Abbaszadeh and Kamal (1989) and Bratvold and Horne (1990), which demonstrated that the analytical solution in the falloff period could be constructed with a reasonable accuracy, by assuming that the total mobility profile does not change during shut-in and using an expression for the rate profile obtained by superposition.

An analytical solution for vertical water injector wells completed in multiple oil zones was proposed by Barreto et al. (2011), and Bela et al. (2018) where the displacement of the two immiscible phases was modeled by a system of partial differential equations.

This work provides a multiple rate approximate solution for pressure response in a water injection well completed in an oil production zone. The proposed formulation was reached by extending and generalizing the existing injection-falloff solution to a general one, considering the total volume of water injected in a reservoir during the test. It will be also shown that all rate periods can be used to estimate parameters related to both water and oil phases.

\section{Theoretical model}

In this work, the gravitational effect and the capillary pressure are neglected. In addition, all calculations assume that the reservoir is subject to the following simplifying hypothesis:

- Homogeneous and isotropic reservoir, with infinite extension;

- Reservoir thickness is constant;

- At the instant $t=0$, reservoir is in equilibrium, i.e., $p(r, t=0)=p_{i}$;
- Water and oil are assumed to be immiscible, slightly compressible fluids with constant viscosity;

- Flow is isothermal;

- Flow is radial and perpendicular to the well section area;

- Rock formation presents a low and constant compressibility;

- Skin damage is neglected;

- Small pressure gradients;

- Non-reactive fluids and rocks;

- Water injection flow rate is constant at the wellbore;

Using the previous hypothesis and following the work of Thompson and Reynolds (1997), the mathematical model can be represented by Eqs. (1)-(4):

$\frac{1}{r} \frac{\partial}{\partial r}\left[\lambda_{\mathrm{t}} r \frac{\partial p}{\partial r}\right]=\frac{\phi c_{\mathrm{t}}}{k} \frac{\partial p}{\partial t}$

$p(r, t=0)=p_{\mathrm{i}}$

$\lim _{r \rightarrow \infty} p(r, t)=p_{\mathrm{i}}$

$-2 \pi k h\left(\lambda_{\mathrm{t}} r \frac{\partial p}{\partial r}\right)_{r_{\mathrm{w}}}=q B_{\mathrm{w}}$.

\section{Multiple rate solution for single-phase flow}

The combination of mass conservation equation, Darcy's law and equations of state, modeling the flow of a fluid through a porous media, provides the solution for a radial infinite reservoir model, assuming single-flow, given by

$p_{\mathrm{i}}-p(r, t)=\Delta p(r, t)=\frac{\alpha_{p} q_{\mathrm{w}} B_{\mathrm{w}}}{h k \hat{\lambda}_{\mathrm{o}}}\left[-\frac{1}{2} E_{i}\left(-\frac{r^{2} \phi \hat{c}_{\mathrm{to}}}{4 \alpha_{t} k \hat{\lambda}_{\mathrm{o}} t}\right)\right]$.

For multiple rates, since this system is linear, and assuming the first flow is zero, the solution can be obtained by using the superposition principle:

$\Delta p\left(r, t_{N}\right)=\frac{\alpha_{p} B}{h k \hat{\lambda}_{\mathrm{o}}} \sum_{j=1}^{n}\left(q_{j}-q_{j-1}\right)\left[-\frac{1}{2} E_{i}\left(-\frac{r^{2} \phi \hat{c}_{\mathrm{to}}}{4 \alpha_{t} k \hat{\lambda}_{\mathrm{o}}\left(t-t_{j-1}\right)}\right)\right]$.

Consider the injection rate given by

$q(r, t)=\frac{h k \hat{\lambda}_{\mathrm{o}}}{\alpha_{p} B} r \frac{\partial p}{\partial r}$.

Combining Eqs. (6) and (7), it is possible to write 
$q(r, t)=r \frac{\partial}{\partial r} \sum_{j=1}^{n}\left(q_{j}-q_{j-1}\right)\left[-\frac{1}{2} E_{i}\left(-\frac{r^{2} \phi \hat{c}_{\mathrm{to}}}{4 \alpha_{t} k \hat{\lambda}_{\mathrm{o}}\left(t-t_{j-1}\right)}\right)\right]$.

Then, the multiple rate expression can be written as

$q(r, t)=\sum_{j=1}^{n}\left(q_{j}-q_{j-1}\right) \mathrm{e}^{-\left[\frac{c_{\mathrm{to}} \mu \phi r^{2}}{4 \alpha_{t} k\left(t-t_{j-1}\right)}\right]}$.

\section{Injection and falloff solution}

In an injectivity test, first occurs the water injection into an oil-saturated zone for a determined period and then occurs a well shut-in and a zero-flow pulse is propagated along the reservoir, characterizing the falloff period. Several reservoir features, such as equivalent permeability and outer boundary condition, might be inferred from the pressure response measured during the test.

Isothermal two-phase flow in radially heterogeneous reservoirs is described by a system of partial differential equations. For small pressure gradients, Darcy's law is satisfied, which is the starting point to compute the pressure variation in the reservoir during an injectivity test.

The approximated solution developed is based on the existing formulation during the injection period and the analytical model for the falloff period in reservoirs presented by Peres et al. (2003). From this work, the approximate analytical solution for the pressure behavior during the water injection period is given by

$$
\begin{aligned}
p_{\mathrm{wf}}-p_{\mathrm{i}}= & \frac{\alpha_{p} q_{\mathrm{w}} B_{\mathrm{w}}}{2 h k \hat{\lambda}_{\mathrm{o}}}\left[\ln \left(\frac{4 \alpha_{t} k \hat{\lambda}_{\mathrm{o}} t}{\mathrm{e}^{\gamma} \widehat{c_{\mathrm{to}}} \phi r_{\mathrm{w}}^{2}}\right)\right] \\
& +\frac{\alpha_{p} q_{\mathrm{w}} B_{\mathrm{w}}}{h k \hat{\lambda}_{\mathrm{o}}}\left[\int_{r_{\mathrm{w}}}^{r_{\mathrm{f}}(t)}\left(\frac{\hat{\lambda}_{\mathrm{o}}}{\lambda_{\mathrm{t}}(r, t)}-1\right) \frac{\mathrm{d} r}{r}\right] .
\end{aligned}
$$

Usually, this equation can be represented by

$\Delta p(t)=\Delta \hat{p}_{0}(t)+\Delta p_{\lambda}(t)$

where $\Delta \hat{p}_{\mathrm{o}}(t)$ represents the single-phase solution for the injection period and the term $\Delta p_{\lambda}(t)$ represents the additional pressure drop due to the mobility contrast between oil and water phases.

The approximate analytical solution for pressure behavior during the falloff period is

$$
\begin{aligned}
\Delta p_{s}(\Delta t)= & \frac{q_{\mathrm{inj}} B \alpha_{p}}{2 k h \hat{\lambda}_{\mathrm{o}}} \ln \left(\frac{t_{p}+\Delta t}{\Delta t}\right) \\
& +\frac{\alpha}{h k \hat{\lambda}_{o}} \int_{r_{\mathrm{w}}}^{r_{\mathrm{f}}(t p)}\left[\frac{\hat{q}_{\mathrm{os}}(r, \Delta t) \hat{\lambda}_{\mathrm{o}}}{\lambda_{\mathrm{t}}\left(r, t_{p}\right)}-\hat{q}_{\mathrm{os}}(r, \Delta t)\right] \frac{\mathrm{d} r}{r} .
\end{aligned}
$$

Analogously to the injection period, the solution in the falloff period can be represented by

$\Delta p_{s}(\Delta t)=\Delta \hat{p}_{\mathrm{os}}(\Delta t)+\Delta p_{\lambda}(\Delta t)$.

\section{Proposed formulation}

Based on the solutions provided by previous studies, the solution for the pressure response for a general case of dualphase flow is the starting point to compute a multiple rate approximate solution.

$$
\begin{aligned}
p_{\mathrm{wf}}-p_{\mathrm{i}}= & \frac{1}{2 \pi h k}\left[\int_{r_{\mathrm{w}}}^{r_{\mathrm{f}}(t)} \frac{q_{\mathrm{inj}}(r, t)}{r \lambda_{\mathrm{t}}(r, t)} \mathrm{d} r-\int_{r_{\mathrm{w}}}^{r_{\mathrm{f}}(t)} \frac{\hat{q}_{\mathrm{o}}(r, t)}{r \hat{\lambda}_{\mathrm{o}}} \mathrm{d} r\right] \\
& +\frac{1}{2 \pi h k}\left[\int_{r_{\mathrm{w}}}^{\infty} \frac{\hat{q}_{\mathrm{o}}(r, t)}{r \hat{\lambda}_{\mathrm{o}}} \mathrm{d} r\right] .
\end{aligned}
$$

In this case, where the rate is variable, the total volume of water injected into the reservoir $v_{\text {inj }}$ at a given instant $t$ can be obtained by

$v_{\text {inj }}(t)=\int_{0}^{t} q_{\text {inj }}(t) \mathrm{d} t$

Equation (15) can be used to estimate the advance of the water displacement front, $r_{\mathrm{f}}(t)$, through the porous medium. In this case, the radial Buckley-Leverett's equation (Buckley and Leverett 1942) is applied, considering $v_{\text {inj }}$

$r_{\mathrm{f}}(t)=\sqrt{r_{\mathrm{w}}^{2}+\frac{B_{\mathrm{w}}}{24 \pi h \phi} f_{\mathrm{w}}^{\prime}\left(S_{\mathrm{w}}\right) \int_{0}^{t} q_{\mathrm{inj}}(t) \mathrm{d} t}$

In this case, for $r<r_{\mathrm{f}}(t)$, the flow in the reservoir $\hat{q}_{\mathrm{o}}(r, t)=q_{\mathrm{inj}}(r, t)$, where $q_{\mathrm{inj}}(r, t)$ can be approximated by the flow superposition provided by Eq. (8). Thus, Eq. (13) can be written as 


$$
\begin{aligned}
p_{\mathrm{wf}}-p_{\mathrm{i}}= & \frac{1}{2 \pi h k \hat{\lambda}_{\mathrm{o}}}\left[\int_{r_{\mathrm{w}}}^{\infty} \frac{\hat{q}_{\mathrm{o}}(r, t)}{r} \mathrm{~d} r\right] \\
& +\frac{1}{2 \pi h k \hat{\lambda}_{\mathrm{o}}}\left[\int_{r_{\mathrm{w}}}^{r_{\mathrm{f}}(t)} q_{\mathrm{inj}}(r, t)\left(\frac{\hat{\lambda}_{\mathrm{o}}}{\lambda_{\mathrm{t}}(r, t)}-1\right) \frac{\mathrm{d} r}{r}\right] .
\end{aligned}
$$

Using the constant fit units to the Brazilian system of measurements, we obtain

$$
\begin{aligned}
p_{\mathrm{wf}}-p_{\mathrm{i}}= & \frac{\alpha_{p}}{h k \hat{\lambda}_{\mathrm{o}}}\left[\left\{\int_{r_{\mathrm{w}}}^{\infty} \frac{\hat{q}_{\mathrm{o}}(r, t)}{r} \mathrm{~d} r\right\}\right. \\
& +\frac{\alpha_{p}}{h k \hat{\lambda}_{\mathrm{o}}}\left\{\int_{r_{\mathrm{w}}}^{r_{\mathrm{f}}(t)} q_{\mathrm{inj}}(r, t)\left(\frac{\hat{\lambda}_{\mathrm{o}}}{\lambda_{\mathrm{t}}(r, t)}-1\right) \frac{\mathrm{d} r}{r}\right\},
\end{aligned}
$$

where $\frac{\alpha_{p}}{h k \hat{\lambda}_{\mathrm{o}}}\left[\int_{r_{\mathrm{w}}}^{\infty} \frac{\hat{\underline{q}}_{\mathrm{o}}(r, t)}{r} \mathrm{~d} r\right]=\Delta \widehat{p_{\mathrm{o}}}$ represents the single-phase solution with multiple rates, computed with properties of the oil phase, given by Eq. (6). On substituting it into Eq. (18), we obtain

$$
\begin{aligned}
p_{\mathrm{wf}}-p_{\mathrm{i}}= & \frac{\alpha_{p} B_{w}}{2 h k \hat{\lambda}_{\mathrm{o}}}\left[\sum_{j=1}^{n}\left(q_{j}-q_{j-1}\right) \ln \left(\frac{4 \alpha_{t} k \hat{\lambda}_{\mathrm{o}}\left(t-t_{j-1}\right)}{\mathrm{e}^{\gamma} \hat{c}_{\mathrm{to}} \phi r_{\mathrm{w}}^{2}}\right)\right] \\
& +\frac{\alpha_{p}}{h k \hat{\lambda}_{\mathrm{o}}}\left[\int_{r_{\mathrm{w}}}^{r_{\mathrm{f}}(t)} q_{\mathrm{inj}}(r, t)\left(\frac{\hat{\lambda}_{\mathrm{o}}}{\lambda_{\mathrm{t}}(r, t)}-1\right) \frac{\mathrm{d} r}{r}\right],
\end{aligned}
$$

which is the proposed multiple rate approximate solution for pressure response in a water injection well, where $q_{\text {inj }}(r, t)$ is given by Eq. (9), using the superposition principle.

\section{Results and discussion}

\section{Comparison between analytical model and numerical model}

First, a comparison was made between the pressure response provided by the proposed formulation and the pressure response provided by the injection and falloff periods for the same reservoir model using a numerical fluid flow simulator (CMG/IMEX). Logtime derivatives for both solutions were compared (Bourdet 2002). These comparisons were made for two different cases: The first considers a mobility ratio greater than 1 , and the second one considers a mobility ratio smaller than 1. Figures 1, 2, 3 and 4 show pressure drop and pressure drop logtime derivative for both cases.

As one can see, in both scenarios the proposed solution represents the expected behavior of logtime derivatives for both injection and falloff periods. When compared to finite difference simulation, some deviations can be observed. We believe that these deviations come from the difference in the computation of water flooding position when comparing the two methods (analytical and numerical). This is worse when mobility ratio is smaller than 1 .
Fig. 1 Mobility ratio $>1-$ injection period

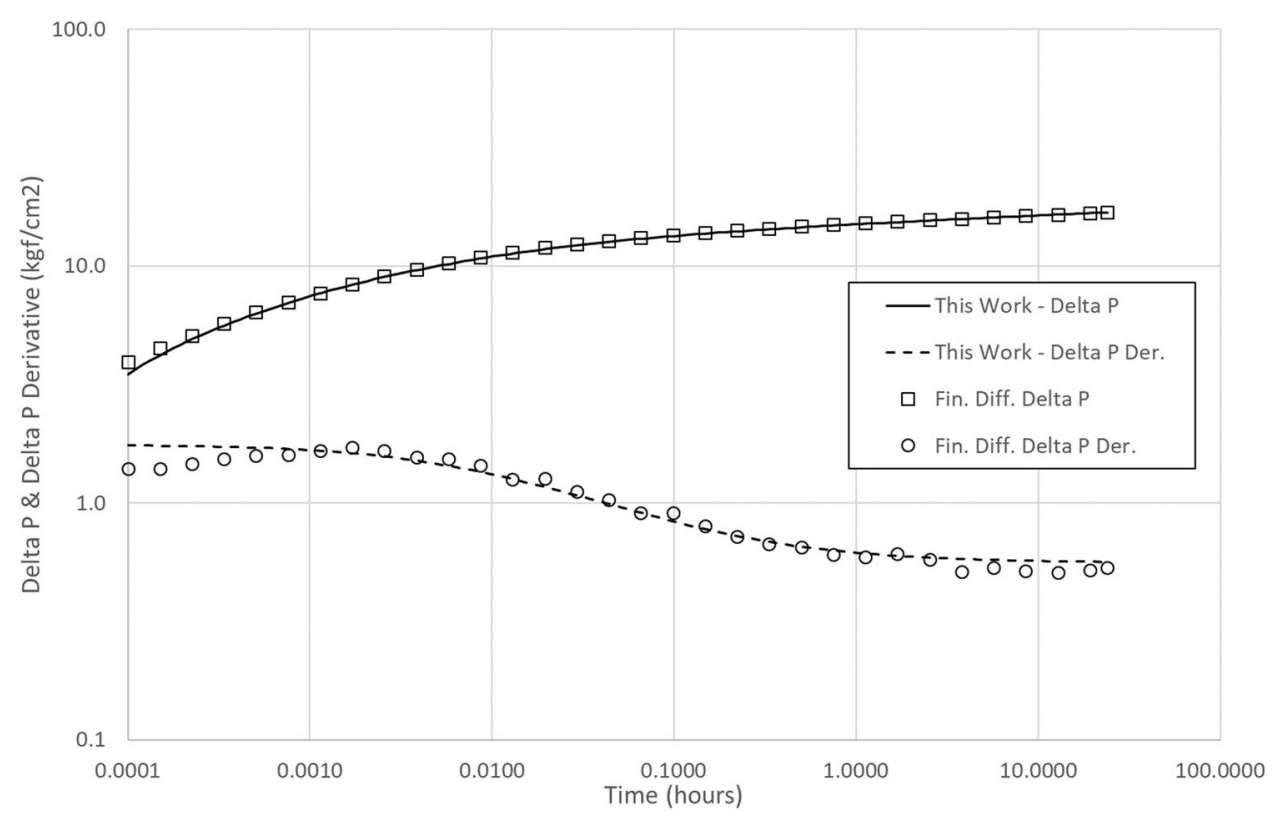




\section{Comparison between the proposed solution and single-phase flow}

It was also analyzed the pressure drop and pressure drop logtime derivative curves, generated by the proposed solution of this work in comparison with the response given by a single-phase flow model (for both phases, oil and water). Figure 5 compares the pressure behavior between a production test (using oil properties and water properties) and an injection test with mobility ratio greater than 1 . As one can see the solution responds as expected: In early times, the injection test logtime derivative responds with the same mobility value of the oil phase. In late times, the value of logtime derivative approximates to water phase mobility.

It can be observed in Fig. 6 (mobility smaller than 1) that the same behavior occurs. Initially, the well bottom pressure logtime derivative computed during the injection period approximates the oil phase logtime derivative. And at the end the injection logtime derivative approximates to water phase mobility.
Fig. 2 Mobility ratio $>1$-falloff period

Fig. 3 Mobility ratio $<1-$ injection period
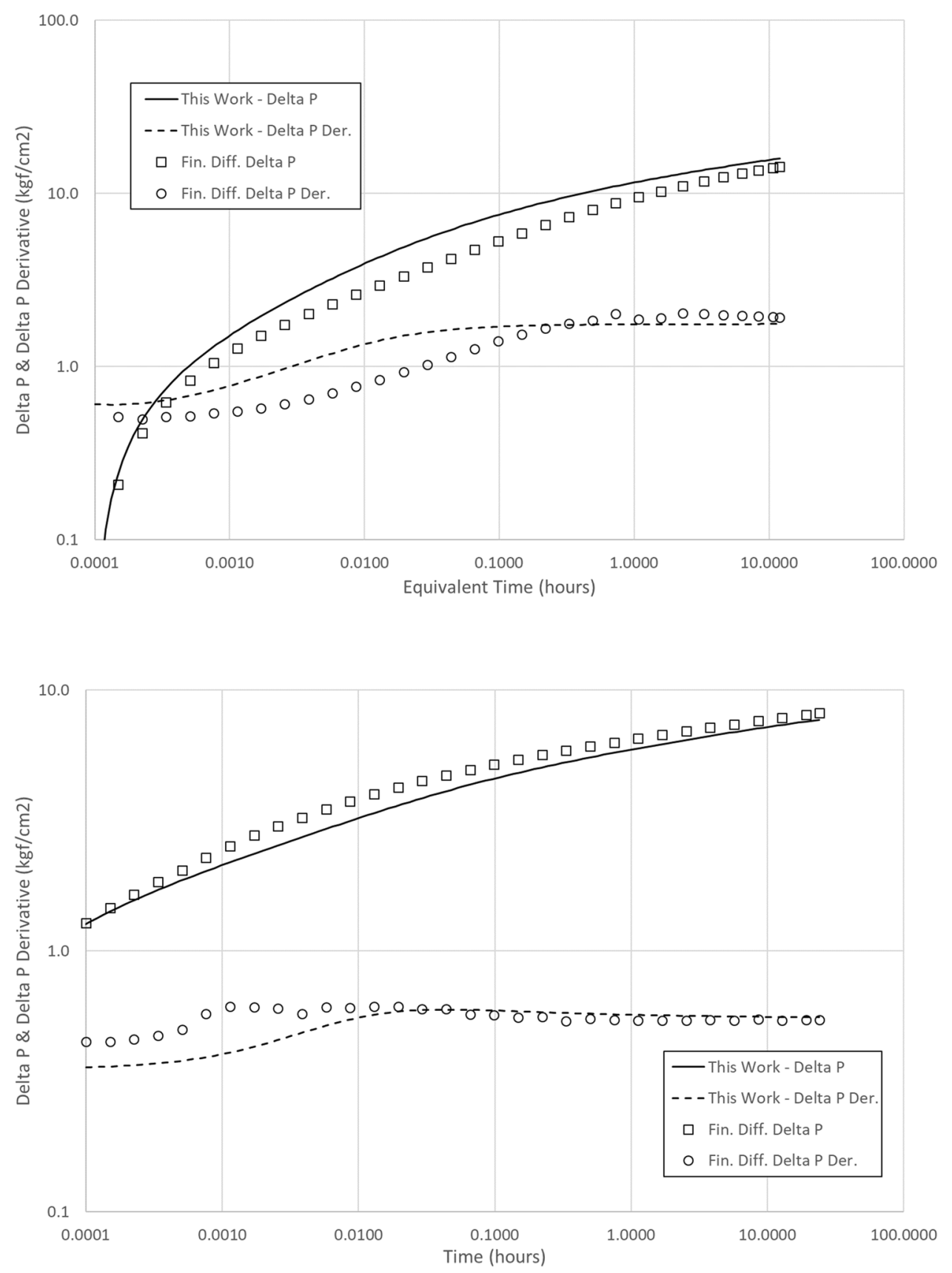
Fig. 4 Mobility ratio $<1$-falloff period
Fig. 5 Comparison between single phase (oil and water properties) and dual phase with mobility ratio $>1$
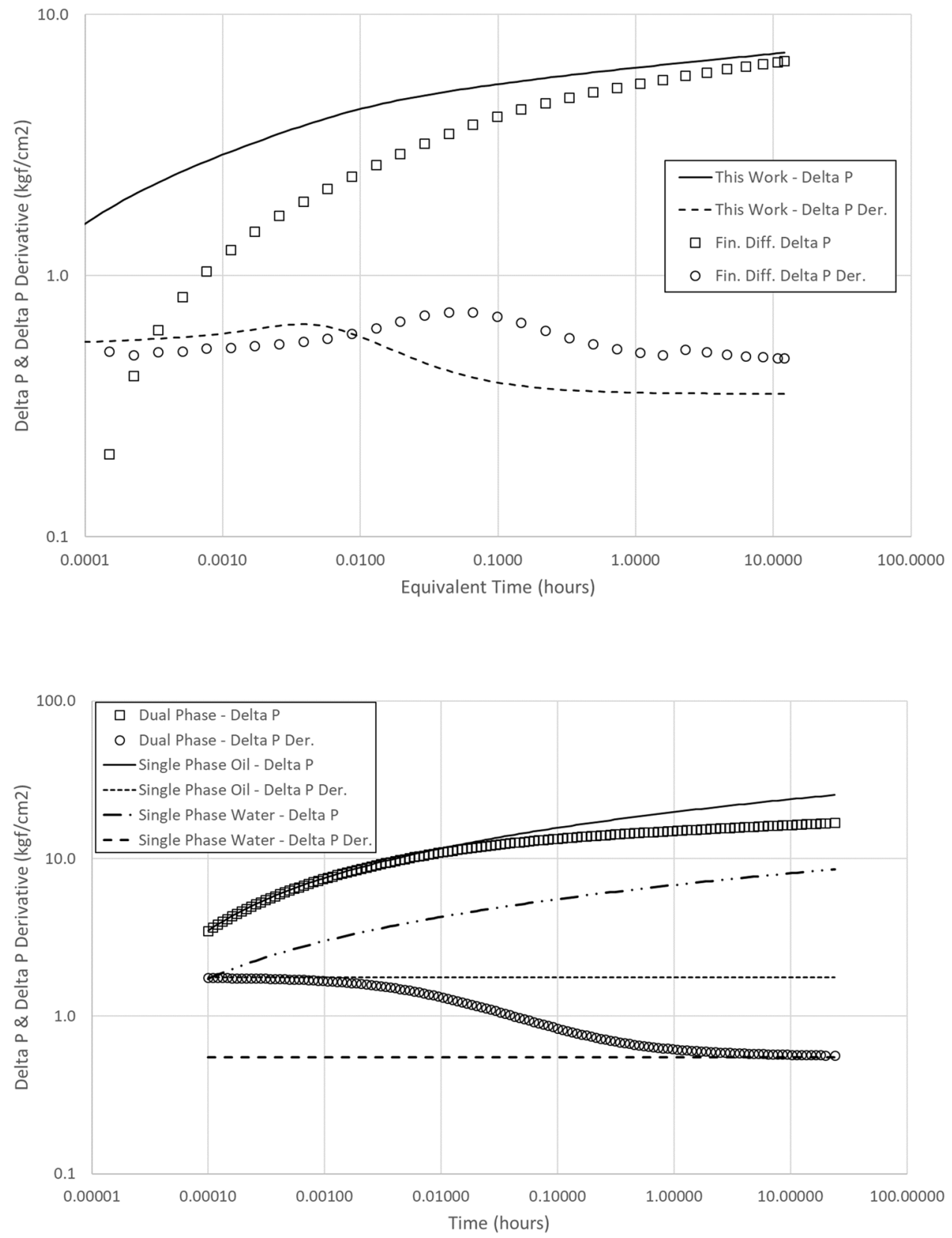

\section{Model with six injection flow rates}

In order to extend the proposed solution for injectivity tests with variable flow rate, a model with six flow rates was performed, whose values are shown in Fig. 7. A comparison with finite difference fluid flow simulator is also plotted to show the accuracy of the proposed formulation. The values for basic rock-fluid properties are shown in Table 1, and the relative permeability curves are plotted in Fig. 8 .

The results of each period are shown in Figs. 9, 10, 11, 12,13 and 14. Normally, the derivatives are plotted against the natural logarithm of time (injection period) or Agarwal's equivalent time (falloff period). In this work, the derivatives for all periods were computed in relation to the natural logarithm of delta time. And instead of using directly the value of pressure drop, we used the normalized pressure drop (pressure drop over injection rate). In this way, all derivatives are comparable. The example is only for mobility ratio greater than one.

The first period represents the pressure response of the first injection rate. As expected, the derivative shape responds in early times approximating the oil mobility and after, at late times, approximating the water mobility. This is a well-known result when an injectivity test is performed. 
Fig. 6 Comparison between single phase (oil and water properties) and dual phase with mobility ratio $<1$

Fig. 7 Well bottom-hole pressure-six injection flow rates scheme
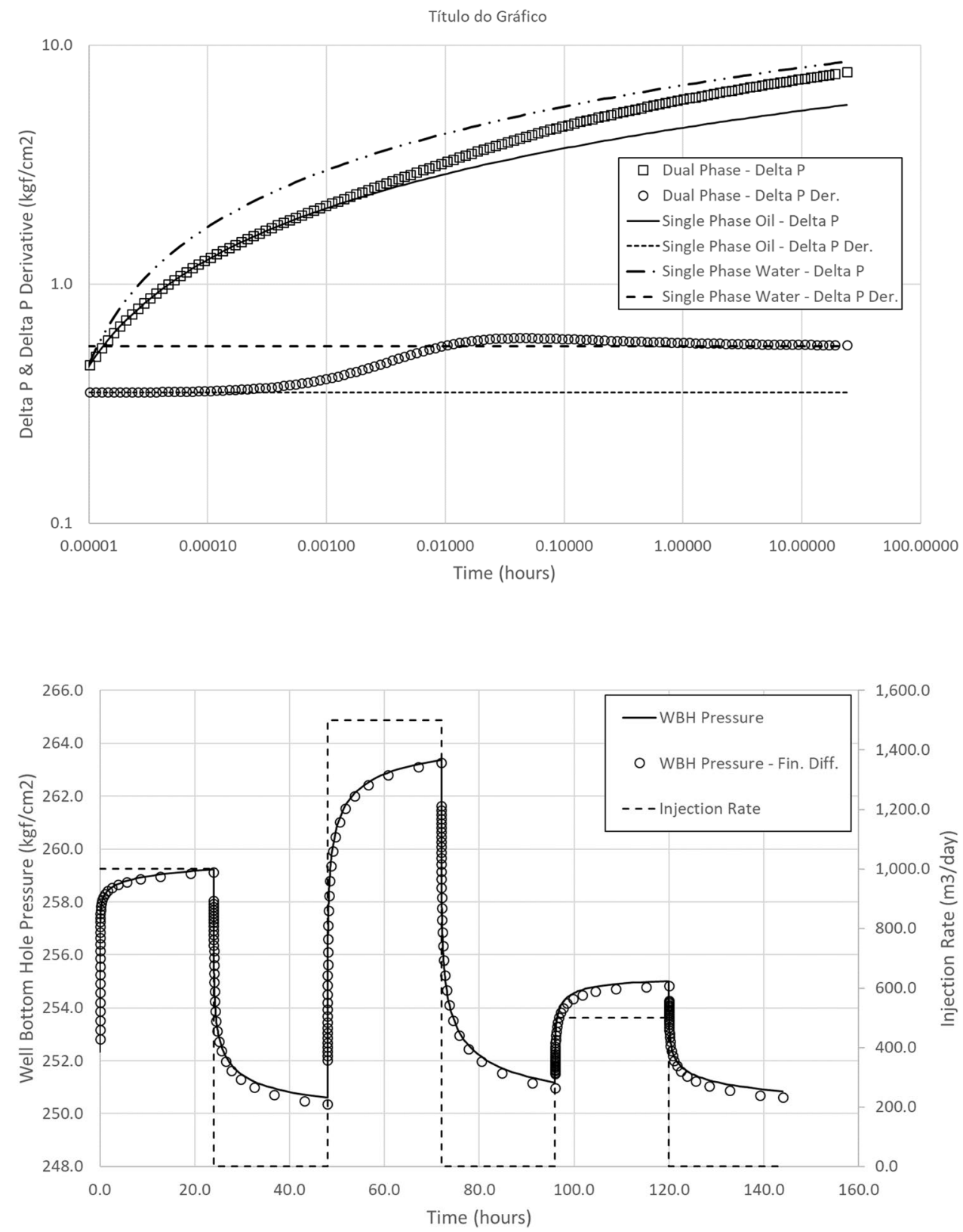

Table 1 Basic rock-fluid properties

\begin{tabular}{lrc}
\hline Property & Value & Unity \\
\hline Permeability $(k)$ & 2000.0 & $\mathrm{mD}$ \\
Porosity $(\phi)$ & 0.2 & \\
Oil viscosity $\left(\mu_{\mathrm{o}}\right)$ & 5.0 & $\mathrm{cp}$ \\
Water viscosity $\left(\mu_{\mathrm{w}}\right)$ & 0.5 & $\mathrm{cp}$ \\
\hline
\end{tabular}

The second period is a zero-rate (or shut-in) period. Again, the expected result is achieved. After the first injection period, a waterfront is created around the wellbore and then the water mobility is firstly observed, followed by the oil mobility at late times. The shape of logtime derivative at the end suggesting that the derivative is tending to zero is due to the derivative computation as explained in the beginning of this section.

All the subsequent periods (third, fourth, fifth and sixth) are $0,1500,0,500$ and $0 \mathrm{~m}^{3} /$ day injection rates, respectively, which are observed followed by the oil mobility at late times. All of them follow the same behavior of the second period, i.e., at early times the solution approximates the water mobility and, at late times, the oil mobility. 
Fig. 8 Relative permeability curves

Fig. 9 First period
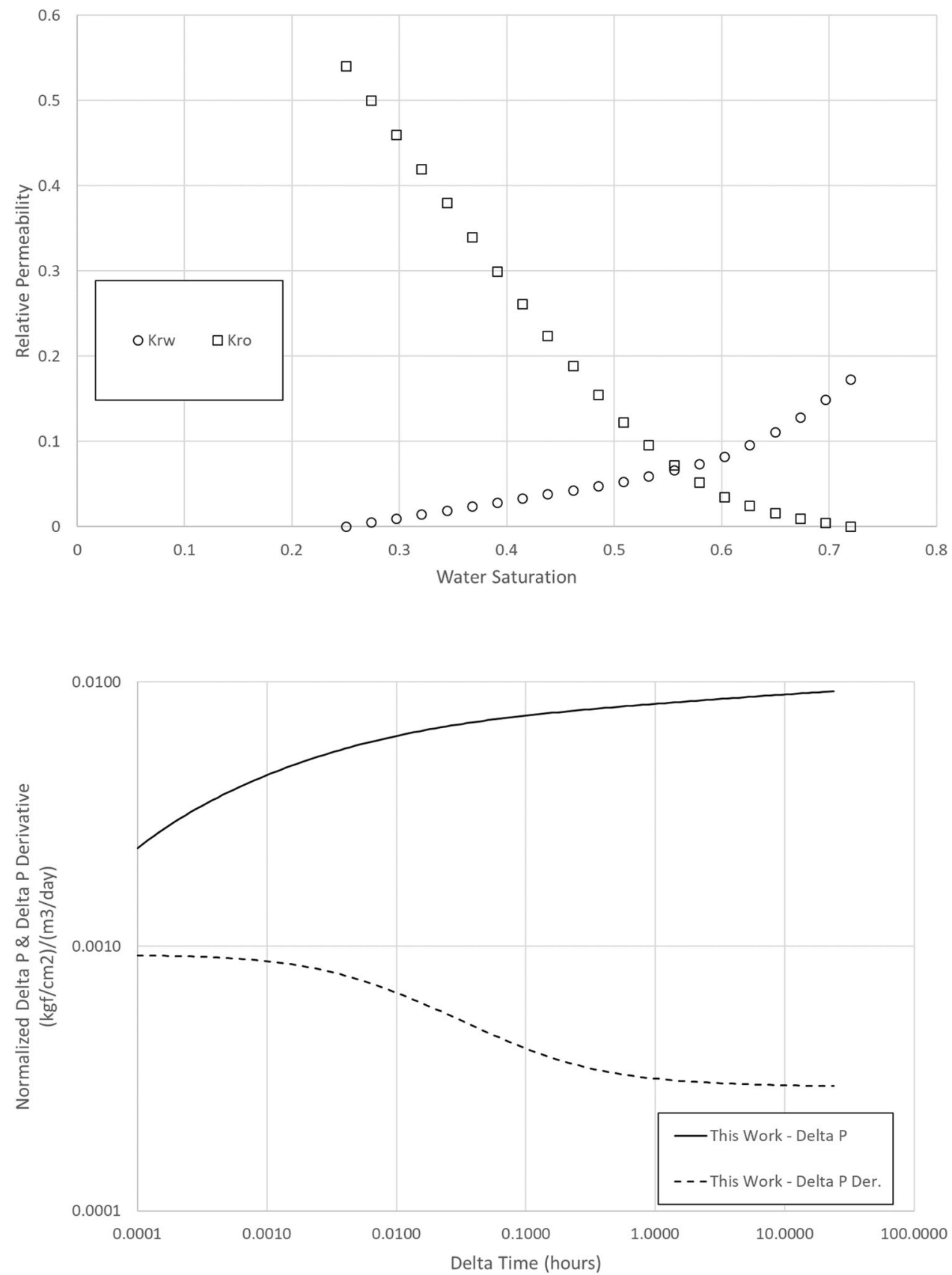

\section{Interpretation}

Another way to validate the proposed solution can be done by comparing the permeability computed or interpreted in the curve of the pressure response with the permeability inserted as reference. For the case of constant flow with single-phase flow, the variation in the bottom well pressure is given by the equation:
$\Delta \hat{p}_{\mathrm{o}}(t)=\frac{\alpha_{p} q_{\mathrm{w}} B_{\mathrm{w}}}{h k \hat{\lambda}_{\mathrm{o}}}\left\{\frac{1}{2} \ln \left(\frac{4 \alpha_{t} k \hat{\lambda}_{\mathrm{o}} t}{\mathrm{e}^{\gamma} \widehat{c_{\mathrm{to}}} \phi r_{\mathrm{w}}^{2}}\right)\right\}$.

This equation shows that a plot of pressures in the wellbore against the natural logarithm of time presents a straight line whose angular coefficient is given by

$m_{\mathrm{o}}=1.151 \frac{\alpha_{p} q_{\mathrm{w}} B_{\mathrm{w}}}{h k \hat{\lambda}_{\mathrm{o}}}$ 
Fig. 10 Second period

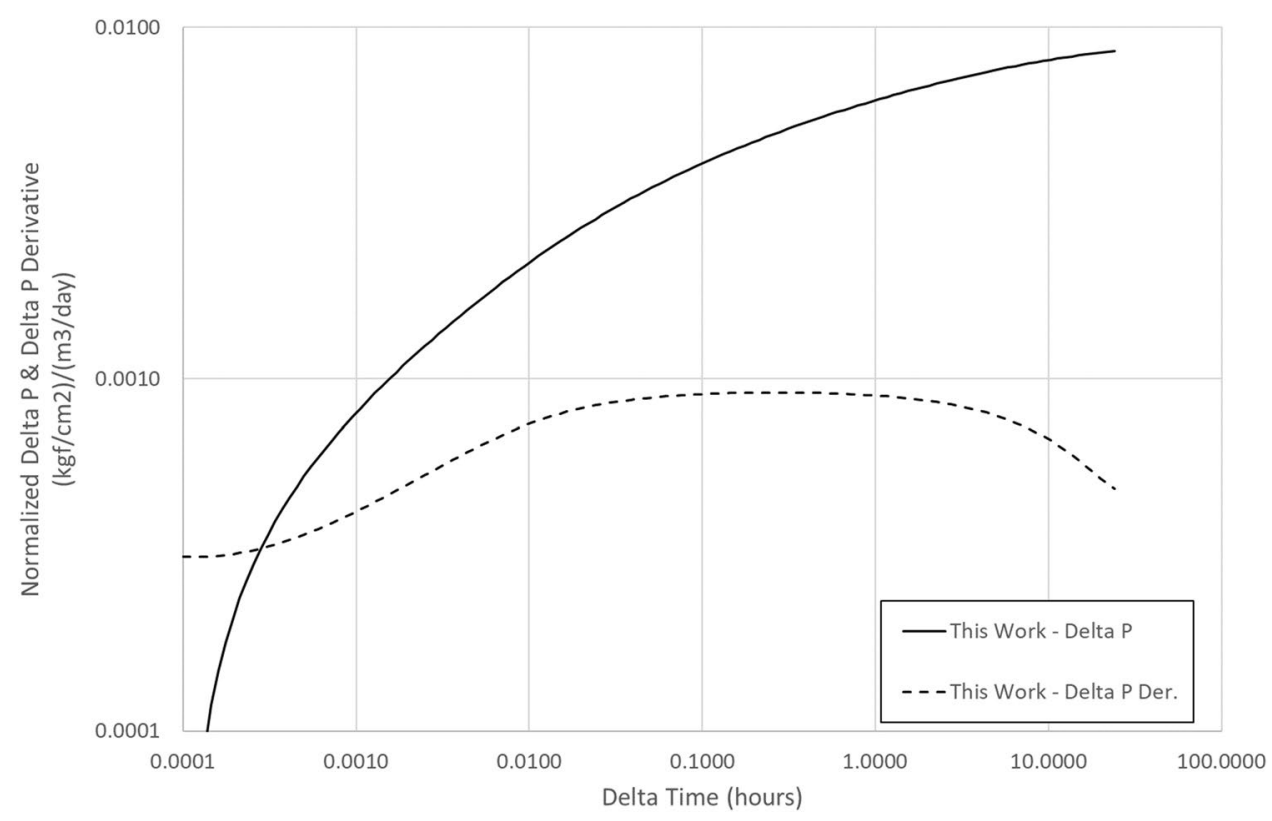

Fig. 11 Third period

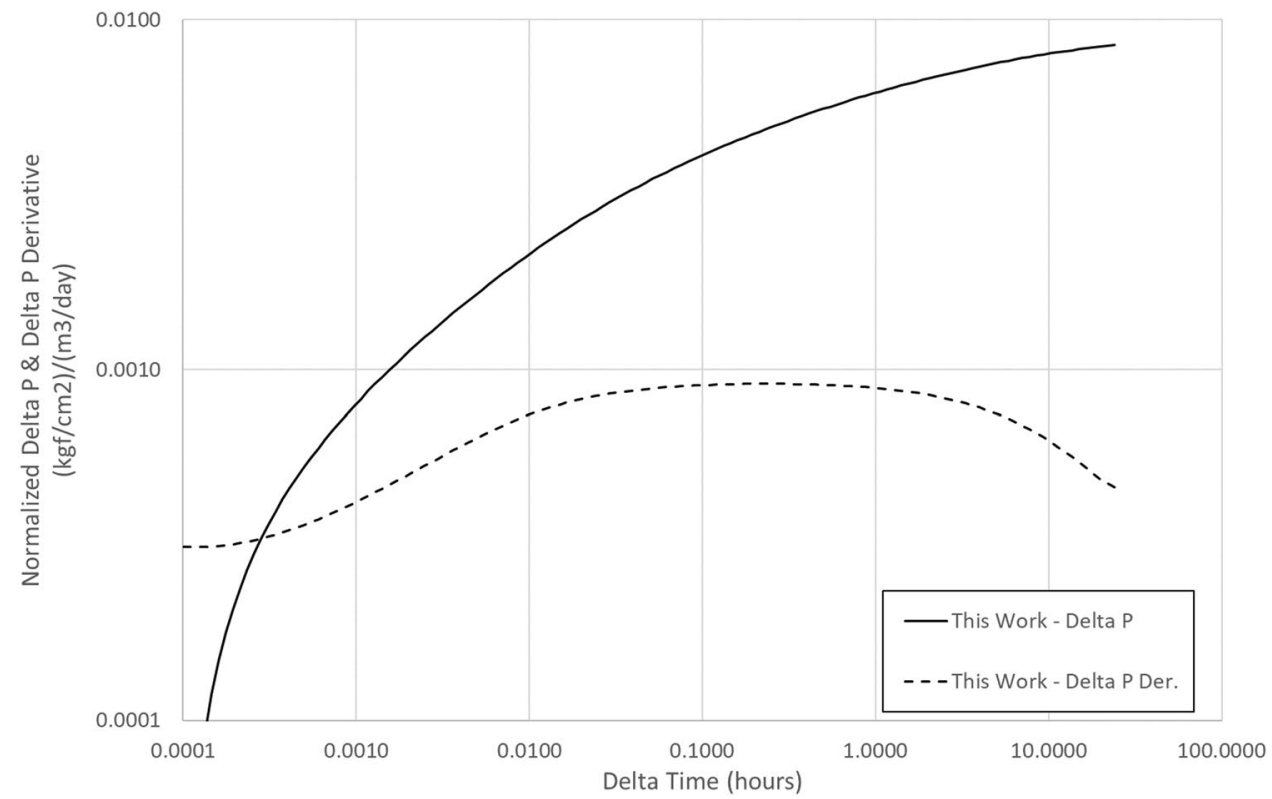

The slope of the line allows computing the reservoir permeability for single-phase flow. For dual-phase flow, as can be seen in derivative curves (Figs. 15 and 16), one can compute two slopes corresponding to both oil and water mobilities. With these values, it is possible to estimate the reservoir permeability using the relative permeability (water and oil) end points.

During the period of water injection into a water reservoir, initially the behavior of the bottom-hole pressure is similar to the case of single-phase oil flow, since the reservoir is mostly saturated with oil, so that by means of the initial inclination, it is possible to obtain the mobility of the oil.

$\hat{\lambda}_{\mathrm{o}}=1.151 \frac{\alpha_{p} q_{\mathrm{w}} B_{\mathrm{w}}}{m_{\mathrm{o}} k h}$.

Over time, the reservoir becomes mostly saturated with water, so that the behavior of the bottom well pressure is similar to the case of single-phase water flow. Thus, by 
Fig. 12 Fourth period

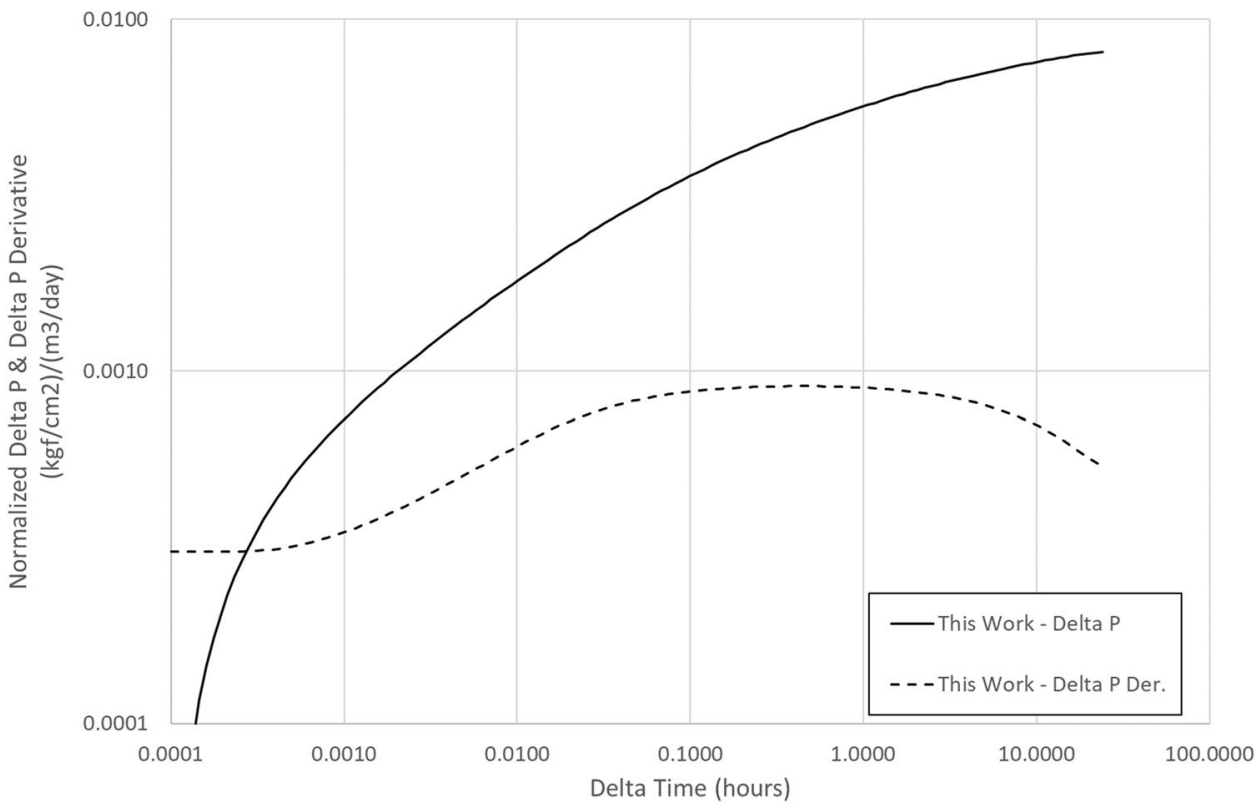

Fig. 13 Fifth period

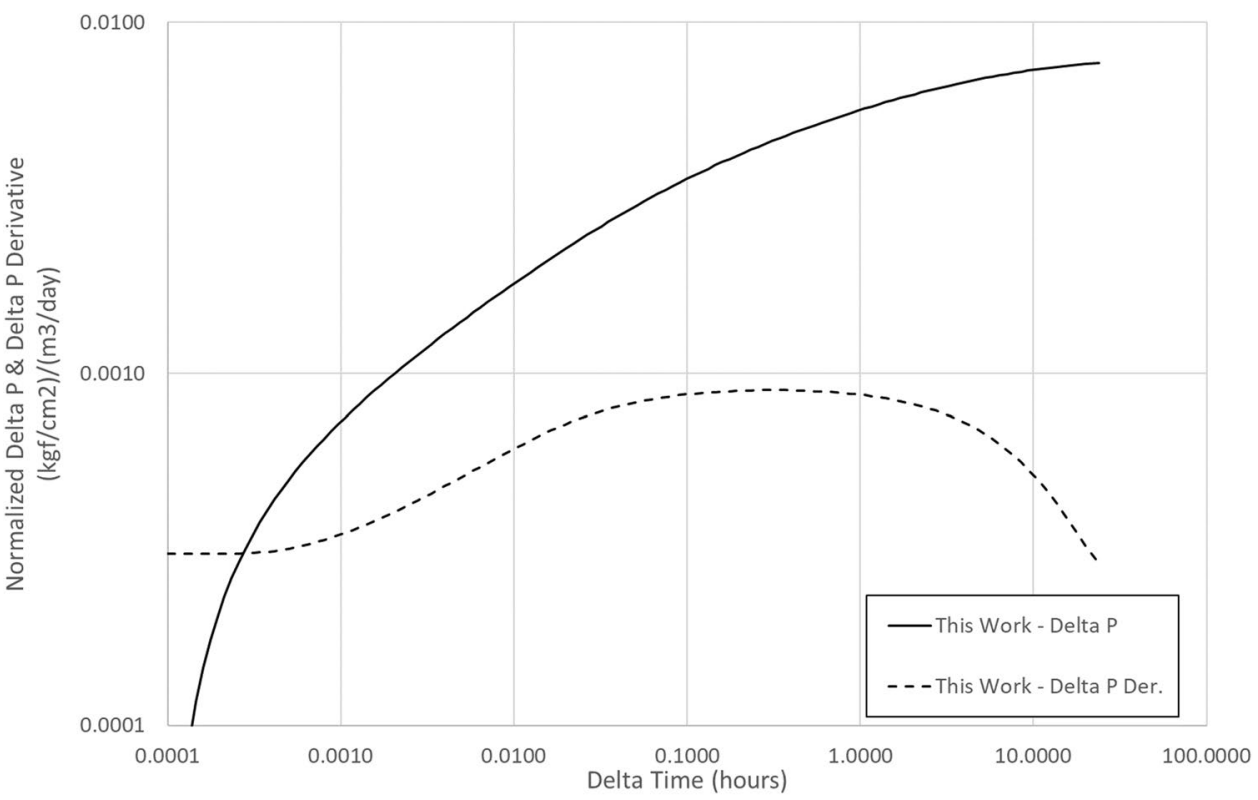

means of the final inclination, it is possible to obtain the mobility of the water.

$\hat{\lambda}_{\mathrm{w}}=1.151 \frac{\alpha_{p} q_{\mathrm{w}} B_{\mathrm{w}}}{m_{\mathrm{w}} k h}$.

So, using the slopes obtained in Fig. 17, it is possible to compute the reservoir permeability for both mobilities (oil and water).

In the same way, using the slopes obtained in Fig. 18, for third and fifth injection periods, it is also possible to compute the reservoir permeability based on water and oil mobilities.

Although the shape of semilog curves of zero-rate periods is different (Fig. 19), the same method can be used to estimate the reservoir permeability based on water and oil mobilities. Table 2 shows the results of reservoir permeability computation compared to reference permeability. As can be seen, the results are very close to all analyzed periods. 
Fig. 14 Sixth period

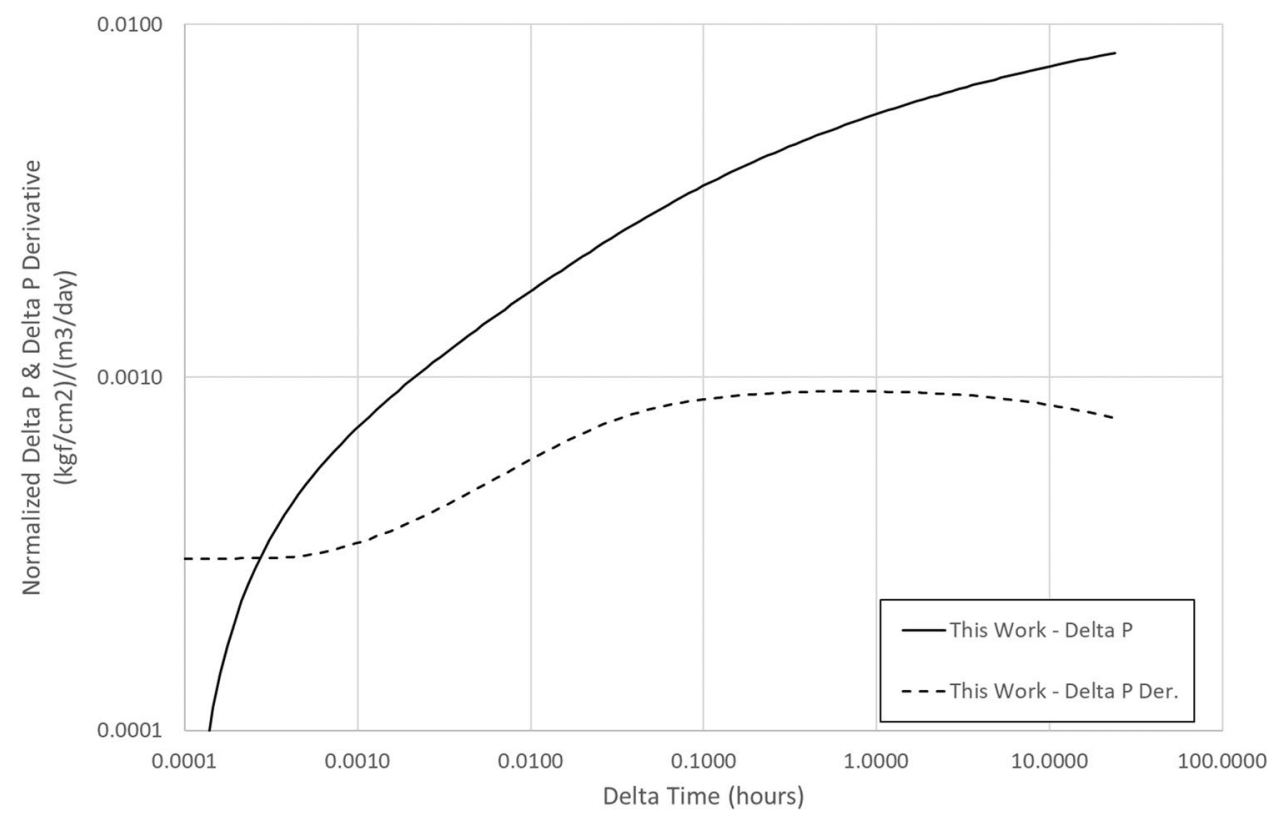

Fig. 15 First period-logtime derivative

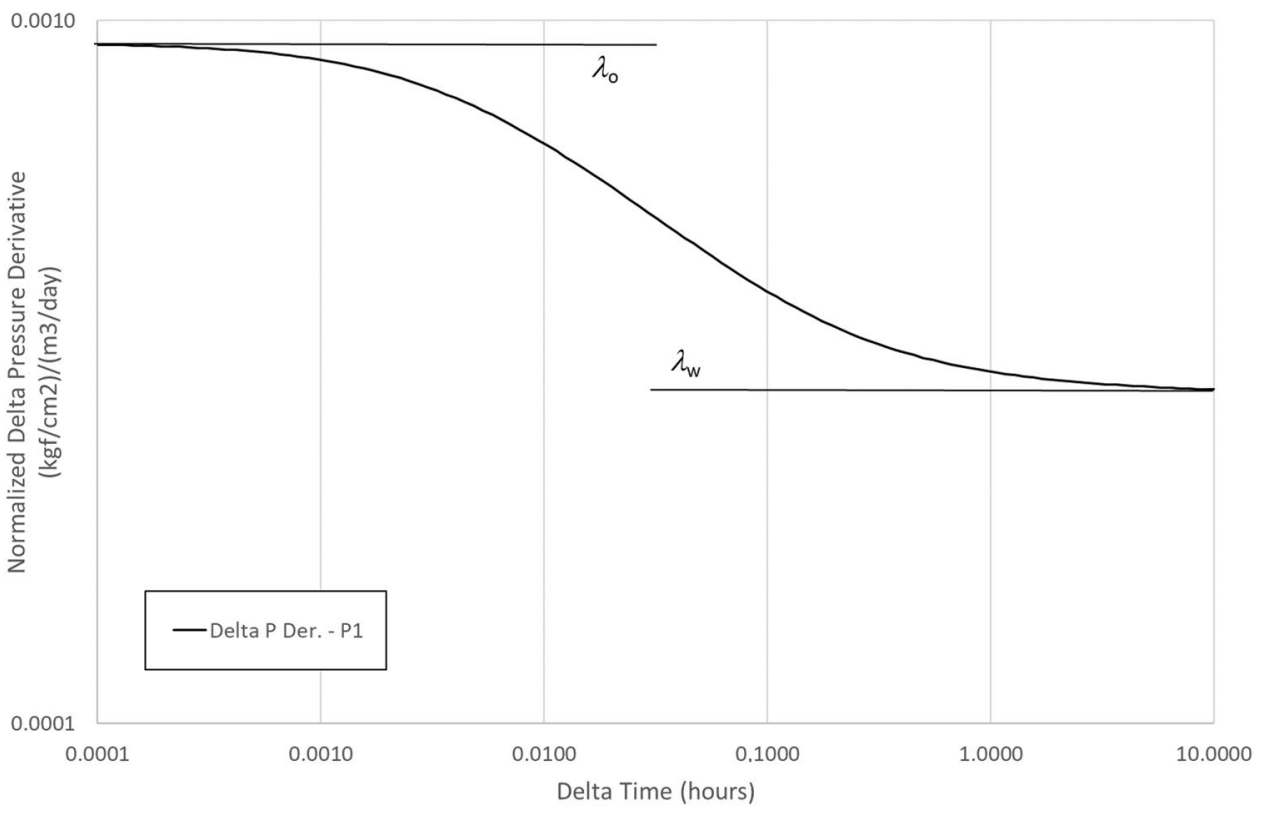

\section{Conclusions and future works}

Based on the results shown here, the following conclusions are presented:

1. An approximate multiple rate approximate solution for pressure response in a water injection well can be obtained with sufficient accuracy, so that it is possible to evaluate the reservoir.
2. Comparison of the proposed solution to a numerical simulator shows a good agreement.

3. It is possible to estimate the reservoir permeability of the reservoir from any period.

4. The presented solution considers a zero-skin factor only.

For future researches, one can wonder that some issues related to injectivity tests can be solved, among others: 
Fig. 16 Five other periodslogtime derivative
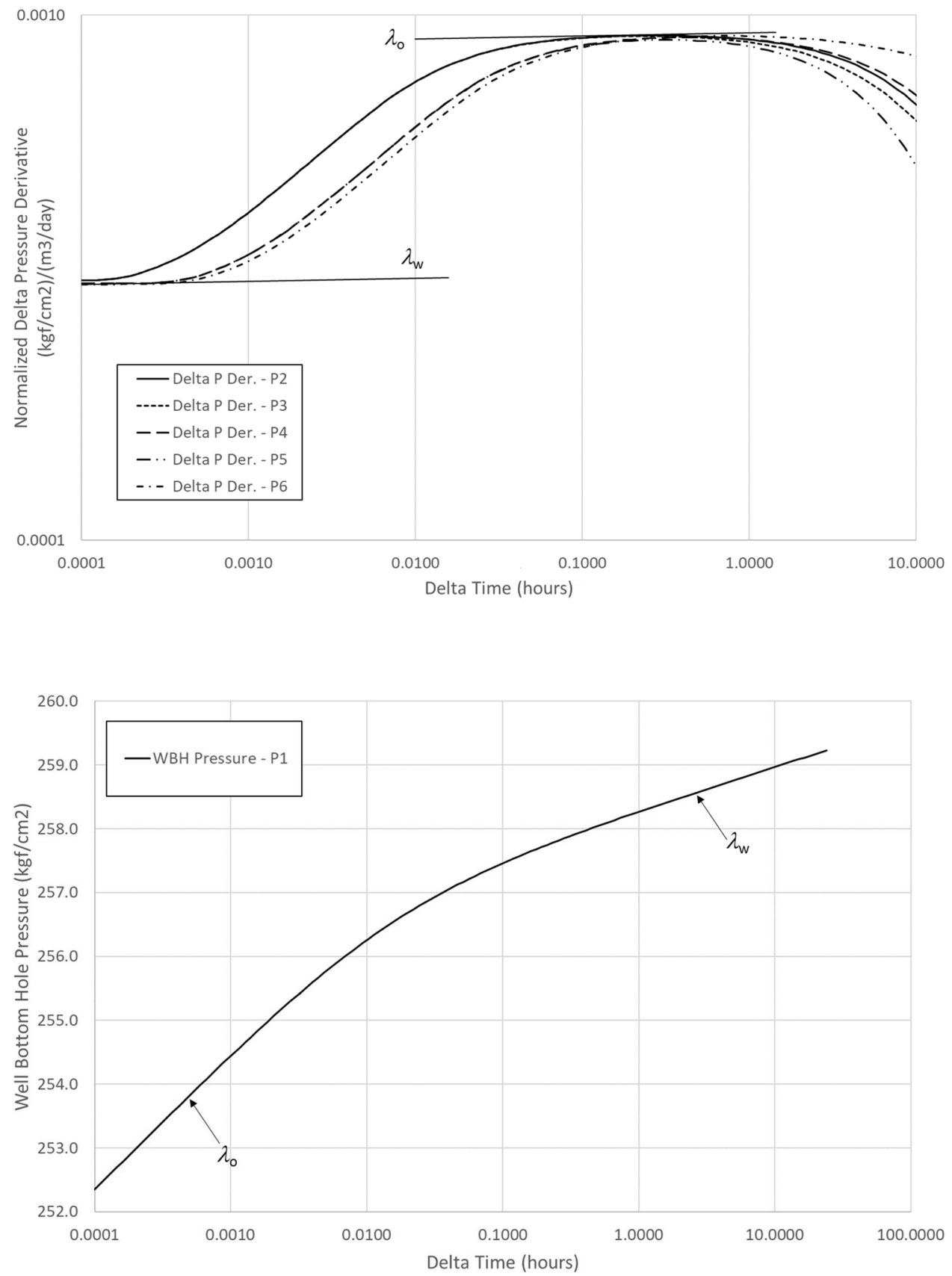

1. Multilayered reservoirs with a multiple rate scheme; this kind of "test" can be observed in oil fields where injector wells are used;
2. Temperature effect; although a lot of work can be found related to temperature effects in pressure behavior, all of them provide solutions for single-phase flow. Here, we have an important issue that can be studied. 
Fig. 18 Third and fifth periods' slopes
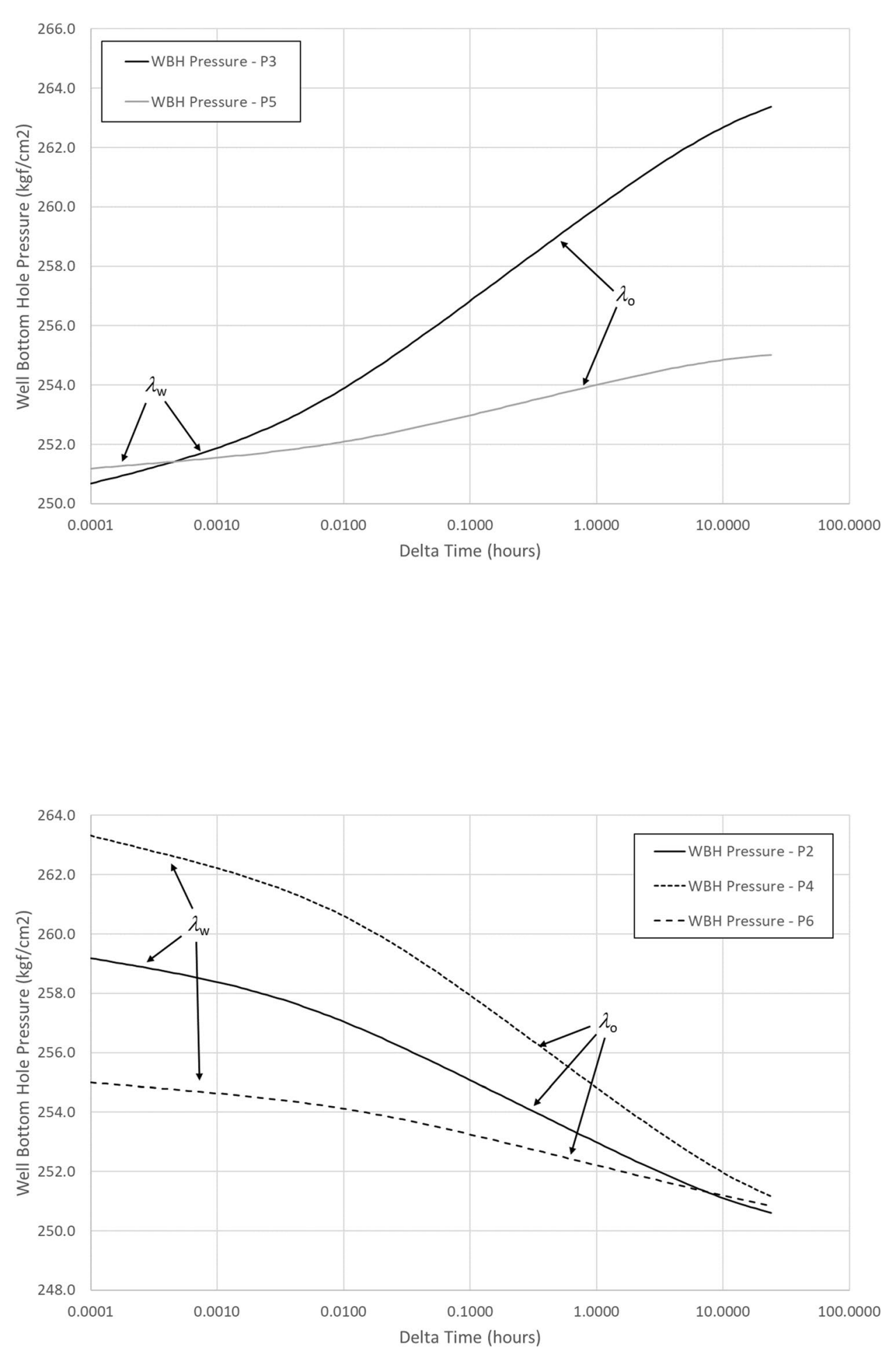

Fig. 19 Second, fourth and sixth zero-rate periods' slopes 
Table 2 Results for oil and water phase

\begin{tabular}{lllllll}
\hline Period & 1 st & 2nd & 3rd & 4th & 5th & 6th \\
\hline$q\left(\mathrm{~m}^{3} /\right.$ day $)$ & 1000.0 & 0.0 & 1500.0 & 0.0 & 500.0 & 0.0 \\
Reference. $K(\mathrm{mD})$ & 2000.0 & 2000.0 & 2000.0 & 2000.0 & 2000.0 & 2000.0 \\
$h(\mathrm{~m})$ & 50.0 & 50.0 & 50.0 & 50.0 & 50.0 & 50.0 \\
Oil & & & & & & \\
$m_{\mathrm{o}}$ & 2.0537 & 2.0994 & 3.1373 & 3.1122 & 1.0271 & 1.0386 \\
$k_{\mathrm{ro}}$ & 0.5403 & 0.5403 & 0.5403 & 0.5403 & 0.5403 & 0.5403 \\
$\mu_{\mathrm{o}}$ & 5.0 & 5.0 & 5.0 & 5.0 & 5.0 & 5.0 \\
$\lambda_{\mathrm{o}}$ & 213.3 & 208.7 & 209.4 & 211.1 & 213.3 & 210.9 \\
Computed $K(\mathrm{mD})$ & 1974.0 & 1931.0 & 1938.2 & 1953.9 & 1973.5 & 1951.7 \\
Rel. error $(\%)$ & 1 & 3 & 3 & 2 & 1 & 2 \\
Water & & & & & & \\
$m_{\mathrm{w}}$ & 0.6825 & 0.7196 & 1.0860 & 1.0633 & 0.3544 & 0.3532 \\
$k_{\mathrm{rw}}$ & 0.1730 & 0.1730 & 0.1730 & 0.1730 & 0.1730 & 0.1730 \\
$\mu_{\mathrm{w}}$ & 0.5000 & 0.5000 & 0.5000 & 0.5000 & 0.5000 & 0.5000 \\
$\lambda_{\mathrm{w}}$ & 641.8 & 608.8 & 605.1 & 618.0 & 618.0 & 620.1 \\
Computed $K(\mathrm{mD})$ & 1855.0 & 1759.5 & 1748.8 & 1786.1 & 1786.1 & 1792.3 \\
Rel. error $(\%)$ & 7 & 9 & 10 & 9 & 9 & 8 \\
\hline
\end{tabular}

Acknowledgements The authors are grateful to ANP for supporting this research. This study was financed in part by the Coordination of Superior Level Staff Improvement-Brasil (CAPES). The authors would like to thank the Master Student Jessica Bittencourt Neto from the Math Department of PUC-Rio, for reviewing the algorithm that did generate all figures of this work.

Open Access This article is licensed under a Creative Commons Attribution 4.0 International License, which permits use, sharing, adaptation, distribution and reproduction in any medium or format, as long as you give appropriate credit to the original author(s) and the source, provide a link to the Creative Commons licence, and indicate if changes were made. The images or other third party material in this article are included in the article's Creative Commons licence, unless indicated otherwise in a credit line to the material. If material is not included in the article's Creative Commons licence and your intended use is not permitted by statutory regulation or exceeds the permitted use, you will need to obtain permission directly from the copyright holder. To view a copy of this licence, visit http://creativecommons.org/licenses/by/4.0/.

\section{References}

Abbaszadeh M, Kamal M (1989) Pressure-transiente testing of water injection wells. SPE-16744-PA Sepere 4(1):115-124

Barreto A Jr, Peres A, Pires A (2011) Water injectivity tests on multilayered oil reservoirs. SPE International, SPE Paper 142746, Macaé, junho de 2011

Bela RV, Pesco S, Barreto A Jr (2018) Modeling falloff tests in multilayer reservoirs. J Petrol Sci Eng 174:161-168

Bourdet D (2002) Well test analysis: the use of advanced interpretatiom models. In: Cubitt J (ed) Handbook of petroleum exploration and production, vol 3. Elsevier, Paris
Bratvolt RB, Horne RN (1990) Analysis of pressure-falloff tests following cold-water injection. SPE 18111-PA SPEFE 5(3):293-302

Buckley SE, Leverett MC (1942) Mechanism of fluid displacement in sands. Trans AIME 146:107-116

Gringarten AC (2005) Analysis of an extended well test to identify connectivity between adjacent compartments in a North Sea reservoir. In: SPE Europec/EAGE annual conference

Oliveira CM, Cordeiro DC, Trevizani AA, Canzian EP, Assunção GG, Romero OJ (2014) Análise paramétrica do deslocamento de óleo em um meio poroso governado pela teoria de Buckley-Leverett. Lat Am J Energy Res 1:82-90

Peres AMM, Reynolds AC (2006) Theory and analysis of injectivity tests on horizontal wells. Soc Petrol Eng SPE J SPE Paper 90907, setembro de 2006

Peres AMM, Boughrara AA, Reynolds AC (2003) Rate superposition for generating pressure falloff solutions. SPE J SPE Paper 84957, junho de 2003

Peres AMM, Boughrara AA, Chen S, Machado AAV, Reynolds AC (2004) Approximate analytical solutions the pressure response at a water injection well. SPE International, SPE Paper 90079, Houston, setembro de 2004

Rosa AJ, Carvalho RS, Xavier JAD (2011) Engenharia de Reservatórios de Petróleo. Editora Interciência, Rio de Janeiro

Thompson LG, Reynolds AC (1997) Well testing for radially heterogeneous reservoirs under single and multiphase flow conditions. SPE Formation Evaluation, SPE Paper 30577, setembro de março de 1997

Publisher's Note Springer Nature remains neutral with regard to jurisdictional claims in published maps and institutional affiliations. 